\title{
Comparison of effect of rectal volume delineation methods on dose constraint endpoints in the treatment of prostate cancer with intensity-modulated radiation therapy
}

\author{
Tony J. C. Wang • Chi Zhang • Ying Wei • David P. Horowitz • \\ Eileen P. Connolly • Simon K. Cheng • Wenzheng Feng • \\ K. S. Clifford Chao • Israel Deutsch
}

Received: 8 March 2013 / Accepted: 12 May 2013 / Published online: 24 May 2013

(C) Springer-Verlag Berlin Heidelberg 2013

\begin{abstract}
Objective Identifying a reliable method of rectal volume delineation (RVD) for prostate cancer treatment planning using intensity-modulated radiation therapy (IMRT) is invaluable for clinical and investigational purposes. We analyzed rectal dose constraint outcomes utilizing commonly used RVD methods. Methods Sixty-two prostate cancer patients treated with IMRT from August 2008 to March 2010 were reviewed. RVD either included the entire rectal volume (ERV) or limited the volume to a $3 \mathrm{~mm}$ rectal wall $(3 \mathrm{MM})$. V50,the percentage of rectal volume receiving $50 \mathrm{~Gy}, \mathrm{~V} 65, \mathrm{~V} 70$, and V75.6 were determined and analyzed for differences. We analyzed factors including definitive and postoperative treatment, rectal volume, and length.

Results For definitive treatment, using ERV, the mean rectal V50, V65, V70, and V75.6 was 41.3, 21.6, 15.1, and 7.0 \%, respectively. Using $3 \mathrm{MM}$, the mean V50, V65, V70, and V75.6 was $37.3,23.4,18.5$, and $10.3 \%$, respectively. The V65, V70, and V75.6 were significantly lower in ERV than $3 \mathrm{MM}(p<0.01)$, while V50 was significantly higher $(p<0.01)$. Similar findings
\end{abstract}

T. J. C. Wang • C. Zhang • D. P. Horowitz • E. P. Connolly •

S. K. Cheng $\cdot$ W. Feng $\cdot$ K. S. C. Chao $\cdot$ I. Deutsch

Department of Radiation Oncology, College of Physicians

and Surgeons, Columbia University, New York, NY, USA

T. J. C. Wang

e-mail: tjw2117@columbia.edu

Y. Wei

Department of Biostatistics, Columbia University,

New York, NY, USA

T. J. C. Wang $\cdot$ I. Deutsch $(\triangle)$

Department of Radiation Oncology, Columbia University,

622 W 168th St., BNH B011,

New York, NY 10032, USA

e-mail: id2182@columbia.edu were noted with rectal volumes $<100 \mathrm{~cm}^{3}$ and length $<10 \mathrm{~cm}$. There was no difference in V50 and V65 with rectal length $\geq 10 \mathrm{~cm}$. For postoperative treatment, using ERV, the mean rectal V50, V65, and V70 was 30.2, 12.9, and $2.7 \%$, respectively. Using $3 \mathrm{MM}$, the mean V50, V65, and V70 was $30.6,16.5$, and $4.7 \%$, respectively. The V65 and V70 were lower in ERV than $3 \mathrm{MM}(p<0.01)$, but not V50. Similar findings were observed with volumes $<100 \mathrm{~cm}^{3}$ and length $\geq 10 \mathrm{~cm}$.

Conclusion We identified different rectal dose-volume endpoints when using common methods of RVD. The differences in dose-volume outcomes are dependent upon prescribed dose, rectal volume, and length. Further analysis, incorporating larger numbers and long-term toxicity outcomes, will contribute to the standardization of contouring and dose-volume analysis for treatment planning.

Keywords Rectal DVH · Prostate cancer · Delineation · IMRT treatment planning

\section{Introduction}

Intensity-modulated radiotherapy (IMRT) is a commonly used treatment modality for prostate cancer. Compared to three-dimensional conformal radiotherapy (3D-CRT), IMRT allows further dose escalation to a target volume, while minimizing normal tissue dose, particularly with concave tumor volumes. The advantage of dose escalation in the treatment of definitive prostate cancer has been demonstrated in numerous studies $[19,20,27]$. Late rectal toxicity, in particular late rectal bleeding, remains an important consideration in IMRT planning [8, 14, 21, 22].

Information has been gleaned from the available studies that provide guidance on the appropriate dose constraints for prostate IMRT to minimize rectal toxicity. Studies have shown 
that keeping the percentage of rectum receiving more than 70 Gy and more than 75 Gy (V70 and V75 Gy) below 25 and $5 \%$, respectively, is predictive of a lower incidence of late bleeding [6, 11, 24]. In addition, limiting the mid-level doses, in the range of the V40-V50, has also been reported to minimize the incidence of bleeding. This benefit was seen, even when patients were treated at relatively low total doses $[2,12,25]$.

The dose volume histogram (DVH), although with limitations, provides us with a reliable way to estimate the risk of normal tissue complications as a function of the dose distribution to tissue volume. However, for "hollow" organs, such as rectum, the dose-wall histogram (DWH) and dosesurface histogram (DSH) have been proposed as alternatives to the entire rectal volume (ERV) of DVH due to the irrelevance of the inner contents to complication risk $[5,15$, 25]. In addition, it has been shown that for a given treatment plan, the DVH of the ERV is different than that of the rectal wall (RW) only $[5,15,17]$. Some studies have reported that the DVH of the RW only appeared more clinically relevant than that of the ERV and was a better fit with the normal tissue complication probability (NTCP) models as well as a predictor of late rectal bleeding with 3D-CRT [13, 23]. However, very limited studies are available for patients treated with IMRT comparing ERV and 3 MM methods in rectal contouring [9]. Although significantly improved, rectal complications remain a significant risk of prostate cancer radiation therapy even in the IMRT era. This side effect can affect the patient's quality of life dramatically. Thus, identifying a reliable method of rectal volume delineation for prostate cancer radiation treatment planning is invaluable for clinical and investigational purposes, especially as IMRT is becoming a routine radiation approach for prostate cancer. Therefore, we conducted the study using dose-volume histograms of patients we treated at our institution and compared the two commonly used methods of rectal volume delineation: ERV and $3 \mathrm{MM}$. We have expanded the study by including a number of patients who received postoperative radiotherapy (RT).

\section{Methods}

\section{Patient selection}

Institutional review board approval was obtained for the study. We identified patients who were treated with IMRT for prostate cancer from August 2008 to March 2010. We excluded patients in whom a rectal balloon was utilized during planning or treatment. The medical records and findings of imaging studies of the remaining 62 patients were reviewed; and in all patients, staging, pretreatment PSA, and Gleason score were analyzed.
Treatment planning

A spiral planning computed tomography (CT) scan was acquired in the supine position, with a slice thickness of $2.5 \mathrm{~mm}$. Patients were immobilized using Vac-Lok system (Civco Medical Solutions, Kalona, Iowa). Patients were required to have an empty bowel and a full bladder. Bowel preparation included taking one bottle of magnesium citrate the night before the simulation. Additionally, a clear liquid diet was recommended to the patients the day before simulation. One Fleet enema was administered by the patient on the morning of the simulation.

Varian Eclipse platform 8.1 (Varian, Palo Alto, California) was utilized for treatment planning. None of the cases required gross tumor volume contours. For definitive cases, the clinical target volume (CTV) included the prostate gland and the seminal vesicles. The volume of seminal vesicle included was dependent upon the level of risk of the prostate disease, with the entire volume of seminal vesicle included for patients with high risk disease. The planning target volume (PTV) was generated with a $1 \mathrm{~cm}$ expansion around the CTV, but limited to $0.5 \mathrm{~cm}$ expansion posteriorly. For postoperative cases, the CTV included the prostate bed, generally as delineated in the consensus guidelines [16]. The PTV was generated with a $1 \mathrm{~cm}$ expansion around the CTV and a $0.6 \mathrm{~cm}$ expansion posteriorly.

The rectum was contoured in two different ways: (1) entire rectal volume including the rectal wall and the rectal lumen and (2) rectal wall based on manual delineation of the outer contour of the rectal wall and the inner contour using a $3 \mathrm{~mm}$ margin. In this instance, the rectal contents were excluded from the rectal contour. For consistency, the rectum was contoured approximately $1 \mathrm{~cm}$ above and below the PTV in the superior-inferior axis.

Treatment was planned for the Varian Trilogy TX linear accelerator with $6 \mathrm{MV}$ photon energy and a high definition multileaf collimator with minimum 0.25 leaf width. Patients received 70.2 Gy for postoperative $(n=18)$ and $\geq 75.6 \mathrm{~Gy}$ (range from 75.6 to $81 \mathrm{~Gy})$ for definitive $(n=44)$ IMRT. Rectal volume was delineated as including the ERV or limiting the volume to a $3 \mathrm{~mm}$ rectal wall ( $3 \mathrm{MM}$ ). For each method of RVD, we determined the percentage of rectal volume receiving 50 Gy (V50), V65, V70, and V75.6. We use two-sided paired $t$ tests to determine if the differences between the two RVD methods in V50, V65, V70, and V75.6 are of statistical significance. The data is stratified, by whether the IMRT was delivered in the definitive or postoperative setting and by volume size and length of the delineated rectum.

Acute toxicity was classified according to the radiation therapy oncology group morbidity grading scale. Late toxicity was not reported, as follow up was short. 
Statistical analyses

All statistical analyses were performed using SPSS software version 20. Two-sided paired $t$ tests were used to determine if the differences between groups are of statistical significance. Only the differences with $P$ values $\leq 0.05$ are considered as statistically significant.

\section{Results}

Forty-four patients were treated definitively, and 18 patients were treated in the postoperative setting. For patients receiving definitive RT, stages $\mathrm{T} 1 \mathrm{c}$ to $\mathrm{T} 2 \mathrm{~b}$ were included, with age ranging from 59 to 82 years old. Four patients received a total dose of $75.6 \mathrm{~Gy}$, one patient received $79.7 \mathrm{~Gy}$, and the rest received $81 \mathrm{~Gy}$. All postoperative RT patients received a total dose of 70.2 Gy in 180 cGy per fraction.

For patients receiving definitive RT, utilizing the ERV method for RVD, outcomes of the mean rectal dose volume for endpoints V50, V65, V70, and V75.6 are 41.3, 21.6, 15.1 , and $7.0 \%$, respectively. Using the $3 \mathrm{MM}$ method, the mean V50, V65, V70, and V75.6 are 37.3, 23.4, 18.5, and $10.3 \%$, respectively. The V65, V70, and V75.6 mean outcomes are significantly higher with the $3 \mathrm{MM}$ method ( $p$ $<0.01)$, while V50 is significantly lower $(p<0.01)$ (Table 1$)$. When stratified by rectal volume and length, similar findings were noted with volumes $<100 \mathrm{~cm}^{3}$ and lengths $<10 \mathrm{~cm}$. However, there was no statistically significant difference in $\mathrm{V} 50$ and V65 with rectal length $\geq 10 \mathrm{~cm}$, but significantly higher V70 and V75.6 were seen with the 3 MM method (Table 2).

For treatment in the postoperative setting, using the ERV method for RVD, outcomes of the mean rectal dose volume for endpoints V50, V65, and V70 are 30.2, 12.9, and 2.7 \%, respectively. Using the $3 \mathrm{MM}$ approach, the mean V50, V65, and V70 are 30.6, 16.5, and $4.7 \%$, respectively. Since the prescribed dose is only $70.2 \mathrm{~Gy}$, V75.6 is $0 \%$ for all patients. The V65 and V70 mean outcomes are significantly higher with the 3 MM method $(p<0.01)$, but V50 does not differ significantly (Table 3 ). When stratified by rectal volume and

Table 1 Comparisons of mean rectal dose volumes in all patients received definitive RT for prostate cancer

\begin{tabular}{llll}
\hline & ERV & $3 \mathrm{MM}$ & $P$ value \\
\hline V50 (\%) & $41.3 \pm 8.1$ & $37.3 \pm 7.0$ & $1.0 \mathrm{E}-10$ \\
V65 (\%) & $21.6 \pm 6.8$ & $23.4 \pm 5.5$ & $5.9 \mathrm{E}-4$ \\
V70 (\%) & $15.1 \pm 5.6$ & $18.5 \pm 5.2$ & $2.1 \mathrm{E}-11$ \\
V75.6 (\%) & $7.0 \pm 5.6$ & $10.3 \pm 5.9$ & $1.2 \mathrm{E}-10$ \\
Max (\%) & $100.8 \pm 2.2$ & $98.5 \pm 13.9$ & 0.27 \\
\hline
\end{tabular}

Table 2 Comparisons of mean rectal dose volumes in definitive RT for prostate cancer stratified by rectal volume and length

\begin{tabular}{|c|c|c|c|c|}
\hline & & ERV & $3 \mathrm{MM}$ & $P$ value \\
\hline \multirow{4}{*}{$\begin{array}{l}\text { Rectal volume } \\
\quad<100 \mathrm{~cm}^{3}\end{array}$} & V50 (\%) & $41.5 \pm 7.8$ & $37.2 \pm 7.1$ & $1.56 \mathrm{E}-7$ \\
\hline & V65 (\%) & $22.2 \pm 7.0$ & $23.3 \pm 6.2$ & 0.03 \\
\hline & V70 (\%) & $15.8 \pm 6.0$ & $18.5 \pm 5.6$ & $6.04 \mathrm{E}-7$ \\
\hline & V75.6 (\%) & $7.5 \pm 5.0$ & $9.9 \pm 6.2$ & $4.1 \mathrm{E}-7$ \\
\hline \multirow{4}{*}{$\begin{array}{l}\text { Rectal volume } \\
\geq 100 \mathrm{~cm}^{3}\end{array}$} & V50 (\%) & $41.1 \pm 9.0$ & $37.5 \pm 7.0$ & 0.03 \\
\hline & V65 (\%) & $20.3 \pm 6.4$ & $23.6 \pm 3.8$ & 0.01 \\
\hline & V70 (\%) & $13.7 \pm 4.7$ & $18.5 \pm 4.3$ & $2.72 \mathrm{E}-6$ \\
\hline & V75.6 (\%) & $6.0 \pm 3.2$ & $11.0 \pm 5.4$ & $1.28 \mathrm{E}-5$ \\
\hline \multirow{4}{*}{$\begin{array}{l}\text { Rectal length } \\
<10 \mathrm{~cm}\end{array}$} & V50 (\%) & $42.3 \pm 4.9$ & $36.7 \pm 5.5$ & $4.1 \mathrm{E}-8$ \\
\hline & V65 (\%) & $22.6 \pm 5.7$ & $24.8 \pm 4.7$ & $4.1 \mathrm{E}-4$ \\
\hline & V70 (\%) & $16.0 \pm 5.1$ & $20.0 \pm 4.5$ & $1.6 \mathrm{E}-9$ \\
\hline & V75.6 (\%) & $7.6 \pm 4.5$ & $11.7 \pm 5.8$ & $1.4 \mathrm{E}-7$ \\
\hline \multirow{4}{*}{$\begin{array}{l}\text { Rectal length } \\
\geq 10 \mathrm{~cm}\end{array}$} & V50 (\%) & $40.0 \pm 11.0$ & $38.1 \pm 8.7$ & 0.055 \\
\hline & V65 (\%) & $20.2 \pm 7.9$ & $21.5 \pm 6.0$ & 0.16 \\
\hline & V70 (\%) & $14.0 \pm 6.3$ & $16.5 \pm 5.4$ & $7.8 \mathrm{E}-4$ \\
\hline & V75.6 (\%) & $6.2 \pm 4.6$ & $8.4 \pm 5.5$ & $6.4 \mathrm{E}-5$ \\
\hline
\end{tabular}

length, similar findings were observed with volumes $<100 \mathrm{~cm}^{3}$ and length $<10 \mathrm{~cm}$, with the exception of V70, which did not show a significant difference (Table 4).

Ten patients treated definitively developed acute grade 1 rectal toxicity ( $22 \%$ ), with remaining patients having no acute rectal toxicity. Seven patients treated postoperatively developed acute grade 1 toxicity (39\%), while all other patients reported no acute rectal toxicity. There were no grade 3 or 4 acute toxicities reported (Table 5). No patients developed late grade 2 or more rectal toxicity in our cohort (Table 6). No correlation could be found between those patients who developed any acute rectal toxicity compared to those who were asymptomatic.

Table 3 Comparisons of mean rectal dose volumes in all patients that received postoperative RT for prostate cancer

\begin{tabular}{llll}
\hline & ERV & $3 \mathrm{MM}$ & $P$ value \\
\hline V50 (\%) & $30.2 \pm 6.1$ & $30.6 \pm 6.0$ & 0.62 \\
V65 (\%) & $12.9 \pm 4.6$ & $16.5 \pm 4.6$ & $8.8 \mathrm{E}-5$ \\
V70 (\%) & $2.7 \pm 2.8$ & $4.7 \pm 4.8$ & 0.0036 \\
V75.6 (\%) & $0.0 \pm 0.0$ & $0.0 \pm 0.0$ & NA \\
Max (\%) & $102.3 \pm 2.7$ & $102.2 \pm 2.7$ & 0.004 \\
\hline
\end{tabular}

$\operatorname{Max}(\%)$ stands for maximal dose received in percentage to prescribed doses. NA not available 
Table 4 Comparisons of mean rectal dose volumes in postoperative RT for prostate cancer stratified by rectal volume and length

\begin{tabular}{|c|c|c|c|c|}
\hline & & ERV & $3 \mathrm{MM}$ & $P$ value \\
\hline \multirow{4}{*}{$\begin{array}{l}\text { Rectal Volume } \\
\quad<100 \mathrm{~cm}^{3}\end{array}$} & V50 (\%) & $31.3 \pm 6.8$ & $31.4 \pm 6.8$ & 0.95 \\
\hline & V65 (\%) & $13.2 \pm 5.4$ & $12.5 \pm 4.9$ & $9.0 \mathrm{E}-4$ \\
\hline & V70 (\%) & $2.1 \pm 2.7$ & $2.7 \pm 3.4$ & 0.02 \\
\hline & V75.6 (\%) & $0.0 \pm 0.0$ & $0.0 \pm 0.0$ & NA \\
\hline \multirow{4}{*}{$\begin{array}{l}\text { Rectal Volume } \\
\geq 100 \mathrm{~cm}^{3}\end{array}$} & V50 (\%) & $27.9 \pm 3.8$ & $29.2 \pm 4.2$ & 0.33 \\
\hline & V65 (\%) & $12.2 \pm 2.6$ & $18.4 \pm 3.3$ & 0.006 \\
\hline & V70 (\%) & $3.95 \pm 2.8$ & $8.7 \pm 4.9$ & 0.005 \\
\hline & V75.6 (\%) & $0.0 \pm 0.0$ & $0.0 \pm 0.0$ & NA \\
\hline \multirow{4}{*}{$\begin{array}{l}\text { Rectal Length } \\
\quad<10 \mathrm{~cm}\end{array}$} & V50 (\%) & $32.3 \pm 6.0$ & $33.3 \pm 5.5$ & 0.35 \\
\hline & V65 (\%) & $13.3 \pm 5.4$ & $17.0 \pm 5.3$ & 0.001 \\
\hline & V70 (\%) & $2.5 \pm 2.9$ & $4.2 \pm 5.2$ & 0.072 \\
\hline & V75.6 (\%) & $0.0 \pm 0.0$ & $0.0 \pm 0.0$ & $\mathrm{NA}$ \\
\hline \multirow{4}{*}{$\begin{array}{l}\text { Rectal Length } \\
\geq 10 \mathrm{~cm}\end{array}$} & V50 (\%) & $26.8 \pm 4.7$ & $26.2 \pm 4.0$ & 0.57 \\
\hline & V65 (\%) & $12.3 \pm 3.3$ & $15.9 \pm 3.5$ & 0.043 \\
\hline & V70 (\%) & $3.0 \pm 2.8$ & $5.6 \pm 4.4$ & 0.024 \\
\hline & V75.6 (\%) & $0.0 \pm 0.0$ & $0.0 \pm 0.0$ & NA \\
\hline
\end{tabular}

$N A$ not available

\section{Discussion}

While IMRT improves normal tissue sparing in treating prostate cancer, lowering doses to the rectum can be a challenge in radiation treatment planning because late rectal bleeding remains one of the major toxicities to consider [1, 26]. Previous studies have shown that in IMRT treatment, as well as 3D-CRT, the method of delineating the rectum significantly influences the dose representation in the DVH. Guckenberger et al. evaluated the dose to the rectum based on dose-volume histogram of ERV, 3 MM, manually delineated rectal wall $(\mathrm{MDWH})$, and the rectal surface (DSH) in treatment planning of the prostate [9, 10]. The delineation of ERV instead of MDWH as organ at risk resulted in significantly superior sparing of the rectum [9]. In IMRT, the differences between dose parameters were larger comparing with 3D-CRT, with significant difference between ERV and all other dose parameters [10]. In addition,

Table 5 Incidence of acute treatment related rectal toxicity after definitive and postoperative intensity-modulated radiation therapy

\begin{tabular}{ll}
\hline & Number (\%) \\
\hline Grade 0 & $45(73 \%)$ \\
Grade 1 & $17(27 \%)$ \\
Grade 2 & 0 \\
Grade 3 & 0 \\
Grade 4 & 0 \\
\hline
\end{tabular}

Table 6 Incidence of late treatment related rectal toxicity after definitive and postoperative intensity-modulated radiation therapy

\begin{tabular}{ll}
\hline & Number (\%) \\
\hline Grade $0-1$ & $62(100 \%)$ \\
Grade 2 or more & $0(0 \%)$ \\
\hline
\end{tabular}

MDWH is not advocated by Guckenberger et al. because the CT density values of the rectal wall often are very similar to the density of the filling, thus a precise delineation of the inner contour of the rectal wall was difficult for some patients, resulting in symmetric rectal wall thicknesses between anterior (high-dose region) and posterior (mid- to low-dose region) rectal wall [9].

We have further studied this subject by comparing specifically the ERV and $3 \mathrm{MM}$ methods in rectal contouring for patients who received IMRT at our institution. We found a consistent significantly superior dose distribution pattern with the ERV method versus the $3 \mathrm{MM}$ method of rectal contouring for both definitive and postoperative IMRT treatment planning in the high-dose region (V65Gy, V70Gy, and V75.6Gy). However, the perceived superior or trends toward superior dose distribution pattern is instead observed in $3 \mathrm{MM}$ method in the intermediate dose region (V50 Gy) in definitive IMRT planning. Interestingly, the same "crossover" patterns of dose distribution are also observed with Guckenberger et al. in their 3D-CRT planning but not in their IMRT planning.

In the postoperative RT cohort, no significant difference was observed at intermediate dose (V50 Gy), but ERV shows superior rectal sparing for V65 and V70 Gy. It is noted that in our study, 70.2 Gy was prescribed to postoperative patients, while 81 Gy to definitive cases. Much lower doses (66 to 73 Gy) were prescribed in the studies from Guckenberger et al. for definitive treatment, and no postoperative IMRT data are available [9, 10]. Our results confirmed the presence of significant difference of DVH results at high-dose range (V65 to V75.6) for different rectal contouring methods, even for patients receiving relatively low dose of radiation, i.e., in postoperative setting.

For patients with a larger rectum (criteria: rectal volume in $\mathrm{ERV} \geq 100 \mathrm{~cm}^{3}$ or rectal length $\geq 10 \mathrm{~cm}$ ) receiving definitive IMRT, consistently significant differences are observed only in V70 and V75.6 Gy but not at lower dose ranges (V50 and V60 Gy) for either criterion. Our results are different from Guckenberger et al., which show no difference in DVH results at all dose ranges. The difference may be at least partly caused by having only very small sample size (ten patients) in their study [10]. For postoperative IMRT patients with distended rectum, results are different, with significant difference observed in V65 and V70 Gy but not at V50 Gy in ERV vs. 3 MM. 
Correlation between Rectal Volume (cm3) vs. CSA (cm2)

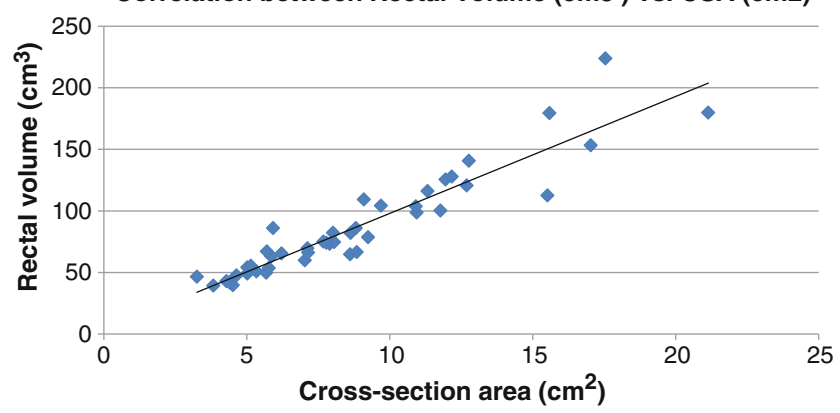

Fig. 1 Correlation between rectal volume (based on ERV) $\left(\mathrm{cm}^{3}\right)$ vs. cross-sectional area (CSA) (rectal volume/rectal length) $\left(\mathrm{cm}^{2}\right)$. High correlational coefficiency is found $(r=0.93)$ indicating patients with large rectal volume have larger CSA

Although a trend has been observed that larger rectal volumes correlate with relatively larger volume differences at the high-dose region (V65 to V75) between ERV and $3 \mathrm{MM}$ methods, only relative low correlation coefficiences between rectal volume and relative volume differences between ERV and $3 \mathrm{MM}$ are seen in our study for V65, V70, and V75.6 Gy ( $r=0.53,0.42$, and 0.51 , respectively). This again indicates that the IMRT benefit for sparing the volume of rectum receiving high dose of radiation is independent of the rectal size/length.

It is interesting to note that V50 Gy is significantly different between ERV and 3 MM methods in RVD in our studies for definitive IMRT treatment planning. The rectal V40-50 has been shown to be an important consideration not only for the prevention of late rectal bleeding, as shown by numerous studies [2, 12, 25], but also for other relatively rare late rectal syndromes such as late rectal incontinence and diarrhea $[4,7,18]$.

In our study, the DVH for ERV appears superior to $3 \mathrm{MM}$ for rectal sparing at high doses. However, it could be associated with higher risk of developing late rectal bleeding, if only rectal wall contouring is more representative of physiologic and anatomical reality. This was suggested by previous studies, which showed $3 \mathrm{MM}$ DWH data being more consistent than ERV when fitting the NTCP models [23].

We have also found that in our definitive IMRT cohort (Fig. 1), rectal volume based on ERV highly correlates with cross-sectional area (CSA) (rectal volume/rectal length) $(r=$ $0.93)$ but does not correlate with rectal length $(r=-0.16)$. Patients with larger rectal volume have less difference in DVHs between ERV and 3 MM methods (Table 2) compared to those with smaller rectal volumes or rectal CSA. Our findings are supported by Guckenberger et al. who demonstrated substantial correlation between DVHs from ERV and $3 \mathrm{MM}$ methods in patients with large rectal volume [9]. It has been reported by de Crevoisier et al. that patients with rectal distension (defined by CSA $>11.2 \mathrm{~cm}^{2}$ ) on the treatment planning CT scan have lower incidence of grade 2 or greater late rectal bleeding [3]. The reason could well be that by using ERV methods, the dose constraints are met similarly to using $3 \mathrm{MM}$ methods in the patients with rectal distension. For patients with small rectal CSA, ERV constraints are much less strict compared to $3 \mathrm{MM}$ methods, indicating the caution of using ERV for patients with small volume rectum, which may have falsely ideal DVH.

\section{Conclusion}

In summary, we identified clear differences in rectal dosevolume histograms when using two common methods of RVD: ERV vs. $3 \mathrm{MM}$. The differences in dose-volume endpoint outcomes are dependent upon prescribed dose and rectal volume and/or length. Further analysis, incorporating larger numbers of patients and long-term toxicity outcomes, will contribute to the standardization of contouring and dose volume analysis for radiation treatment planning.

Acknowledgments The authors gratefully acknowledge the assistance of Jack Kessel for the dosimetry review of this manuscript.

Conflict of interest Tony J. C. Wang, Chi Zhang, Ying Wei, David P. Horowitz, Eileen P. Connolly, Simon K. Cheng, K.S. Clifford Chao, and Israel Deutsch declare that they have no conflict of interest.

Ethical Statement This article does not contain any studies with human subjected and performed upon by any of the authors.

\section{References}

1. Cahlon O et al (2008) Ultra-high dose (86.4 Gy) IMRT for localized prostate cancer: toxicity and biochemical outcomes. Int J Radiat Oncol Biol Phys 71:330-337

2. Cozzarini C et al (2003) Significant correlation between rectal DVH and late bleeding in patients treated after radical prostatectomy with conformal or conventional radiotherapy (66.6-70.2 Gy). Int J Radiat Oncol Biol Phys 55:688-694

3. de Crevoisier R et al (2005) Increased risk of biochemical and local failure in patients with distended rectum on the planning ct for prostate cancer radiotherapy. Int J Radiat Oncol Biol Phys 62:965-973

4. Fiorino $\mathrm{C}$ et al (2008) Clinical and dosimetric predictors of late rectal syndrome after 3D-CRT for localized prostate cancer: preliminary results of a multicenter prospective study. Int $\mathrm{J}$ Radiat Oncol Biol Phys 70:1130-1137

5. Fiorino C, Gianolini S, Nahum AE (2003) A cylindrical model of the rectum: comparing dose-volume, dose-surface, and dose-wall histograms in the radiotherapy of prostate cancer. Phys Med Biol 48:2603-2616

6. Fiorino C et al (2009) Dose-volume effects for normal tissues in external radiotherapy: pelvis. Radiother Oncol 93:153-167

7. Fonteyne $\mathrm{V}$ et al (2007) Late radiotherapy-induced lower intestinal toxicity (RILIT) of intensity-modulated radiotherapy for prostate cancer: the need for adapting toxicity scales and the appearance of the sigmoid colon as co-responsible organ for lower intestinal toxicity. Radiother Oncol 84:156-163 
8. Ghadjar P et al (2008) Acute and late toxicity in prostate cancer patients treated by dose escalated intensity modulated radiation therapy and organ tracking. Radiat Oncol 3:35

9. Guckenberger $\mathrm{M}$ et al (2006) Distinct effects of rectum delineation methods in 3D-conformal vs. IMRT treatment planning of prostate cancer. Radiat Oncol 1:34

10. Guckenberger $M$ et al (2006) Influence of rectum delineation (rectal volume vs. rectal wall) on IMRT treatment planning of the prostate. Strahlenther Onkol 182:721-726

11. Huang EH et al (2002) Late rectal toxicity: dose-volume effects of conformal radiotherapy for prostate cancer. Int J Radiat Oncol Biol Phys 54:1314-1321

12. Jackson A et al (2001) Late rectal bleeding after conformal radiotherapy of prostate cancer. Ii. volume effects and dosevolume histograms. Int J Radiat Oncol Biol Phys 49:685-698

13. Koper PC et al (2004) Impact of volume and location of irradiated rectum wall on rectal blood loss after radiotherapy of prostate cancer. Int J Radiat Oncol Biol Phys 58:1072-1082

14. Leibel SA et al (2003) Technological advances in external-beam radiation therapy for the treatment of localized prostate cancer. Semin Oncol 30:596-615

15. Li S et al (1997) Analysis of the dose-surface histogram and dosewall histogram for the rectum and bladder. Med Phys 24:1107-1116

16. Michalski JM et al (2010) Development of rtog consensus guidelines for the definition of the clinical target volume for postoperative conformal radiation therapy for prostate cancer. Int $\mathbf{J}$ Radiat Oncol Biol Phys 76:361-368

17. Onal C et al (2009) Comparison of rectal volume definition techniques and their influence on rectal toxicity in patients with prostate cancer treated with 3D conformal radiotherapy: a dosevolume analysis. Radiat Oncol 4:14
18. Peeters ST et al (2006) Rectal bleeding, fecal incontinence, and high stool frequency after conformal radiotherapy for prostate cancer: normal tissue complication probability modeling. Int $\mathrm{J}$ Radiat Oncol Biol Phys 66:11-19

19. Pollack A et al (2004) Prostate cancer radiotherapy dose response: an update of the fox chase experience. J Urol 171:1132-1136

20. Pollack A et al (2002) Prostate cancer radiation dose response: results of the $\mathrm{m}$. D. Anderson phase III randomized trial. Int J Radiat Oncol Biol Phys 53:1097-1105

21. Schultheiss TE et al (1997) Late gi and gu complications in the treatment of prostate cancer. Int J Radiat Oncol Biol Phys 37:3-11

22. Skwarchuk MW et al (2000) Late rectal toxicity after conformal radiotherapy of prostate cancer (1): multivariate analysis and doseresponse. Int J Radiat Oncol Biol Phys 47:103-113

23. Tucker SL et al (2004) Comparison of rectal dose-wall histogram versus dose-volume histogram for modeling the incidence of late rectal bleeding after radiotherapy. Int J Radiat Oncol Biol Phys 60:1589-1601

24. van der Laan HP et al (2008) Grading-system-dependent volume effects for late radiation-induced rectal toxicity after curative radiotherapy for prostate cancer. Int J Radiat Oncol Biol Phys 70:1138-1145

25. Wachter $\mathrm{S}$ et al (2001) Rectal sequelae after conformal radiotherapy of prostate cancer: dose-volume histograms as predictive factors. Radiother Oncol 59:65-70

26. Zelefsky MJ et al (2000) Clinical experience with intensity modulated radiation therapy (IMRT) in prostate cancer. Radiother Oncol 55:241-249

27. Zelefsky MJ et al (2001) High-dose radiation delivered by intensity modulated conformal radiotherapy improves the outcome of localized prostate cancer. J Urol 166:876-881 